\title{
¿S Research Square

\section{Hydrothermal Synthesis of Silver Decorated Reduced Graphene Oxide (rGO) Nanoflakes with effective Photocatalytic Activity for Wastewater Treatment}

\section{Muhammad Ikram ( $\nabla$ dr.muhammadikram@gcu.edu.pk)}

Government College University Lahore https://orcid.org/0000-0001-7741-789X

\section{Ali Raza}

Riphah International University - Lahore Campus

Muhammad Imran

University of the Chinese Academy of Sciences

\section{Anwar Ul-Hamid}

King Fahd University of Petroleum \& Minerals

\section{Atif Shahbaz}

Government College University Lahore Faculty of Science and Technology

\section{Salamat Ali}

Riphah International University - Lahore Campus

\section{Nano Express}

Keywords: Silver, nanoflake, rGO, photocatalytic, waste water

Posted Date: March 30th, 2020

DOI: https://doi.org/10.21203/rs.2.23414/v3

License: (c) (1) This work is licensed under a Creative Commons Attribution 4.0 International License.

Read Full License

Version of Record: A version of this preprint was published at Nanoscale Research Letters on April 28th, 2020. See the published version at https://doi.org/10.1186/s11671-020-03323-y. 


\section{Abstract}

Graphene oxide (GO) was obtained through modified hummers method and reduced graphene oxide (rGO) was acquired by employing heat treatment. Various concentrations (2.5, 5, 7.5 and $10 \mathrm{wt}$ \%) of silver (Ag) were incorporated in GO nanosheets by adopting hydrothermal approach. Synthesized Ag decorated $\mathrm{rGO}$ photocatalyst $\mathrm{Ag} / \mathrm{rGO}$ was characterized using X-ray diffraction (XRD) to determine phase purity and crystal structure. XRD patterns showed the formation of $\mathrm{GO}$ to $\mathrm{Ag} / \mathrm{rGO}$. Molecular vibration and functional groups were determined through Fourier Transform Infrared spectroscopy (FTIR). Optical properties and a decrease in bandgap with insertion of Ag were confirmed with UV-visible (Uv-vis.) spectrophotometer and Photoluminescence (PL). Electronic properties and disorders in carbon structures were investigated through Raman spectroscopy that revealed the existence of characteristic bands ( $D$ and G). Surface morphology of prepared samples was examined with Field Emission Scanning Electron Microscope (FESEM). Homogeneous distribution, size and spherical shape of Ag NPs over rGO sheets were further confirmed with the help of High-Resolution Transmission Electron Microscope (HR-TEM). Dye degradation of doped and undoped samples was examined through Uv-vis. spectra. Experimental results indicated that photocatalytic activity of Ag@rGo enhanced with increased doping ratio owing to diminished electron-hole pair recombination. Therefore, it is suggested that Ag@rGO can be used as a beneficial and superior photocatalyst to clean environment and wastewater.

\section{Introduction}

Water on earth is akin to blood in our bodies. It is a key resource material for survival and development of all living species. Albeit $71 \%$ of earth's surface is covered with water, only $0.03 \%$ of total water is considered as freshwater that can be directly utilizable by humans through freshwater lakes, rivers and shallow groundwater [1]. In recent decades, inadequate availability of clean drinking water has presented itself as an unrelenting global concern. Rapid growth of world's population and industrialization has led to increasing environmental pollution, such that around 750 million people face lack of access to clean water [2,3]. Water reservoirs are recurrently contaminated by various hazardous pollutants containing heavy metal ions, dyes, oil and other chemicals that are released from different leather tanneries and industries related to textile, rubber, paper, cosmetics, dyeing, plastic and food [4]. According to the World Bank report, $17-20 \%$ of water pollution is instigated by textile industry. Annually $~ 1 / 10$ th million types of dyes are produced in numerous textile processes, among these dyes methylene blue (MB) $10-15 \%$ is directly released in the effluent. These pollutants create serious health issues such as cancer, skin irascibilities, allergy, and liver malfunctioning and are also harmful to aquatic life [4, 5].

To address these global problems, certain conventional treatment approaches such as ion exchange, electrolysis, carbon filter, chemical coagulation, biological methods, membrane filtration and reverse osmosis (RO) are employed. Nevertheless, series of drawbacks and limitations are associated with these techniques including ineptness, complex procedure, high sludge formation, high implementation and operational cost and use of large amounts of energy $[4,6,7]$. Thus, efficient technologies with 
aforementioned properties need to be developed; among such techniques, photocatalysis overcomes maximum deficiencies.

To date, photocatalytic degradation using inorganic semiconductor nanomaterials has exhibited vast desirability and interest for researchers owing to its excellent physical and chemical properties such as low toxicity, electrochemical stability, super oxidative capacity, cost effectiveness and environmental viability $[2,8,9]$. During the photocatalytic (PC) process, nanomaterials absorb greater visible light energy than bandgap initiated excitation between valence and conduction bands. Through charge separation, electron-hole pairs are generated. Free radicals $(\mathrm{OH})$ oxidize organic compounds and degrade contaminants $[8,10]$.

On the other hand, some crucial factors are vital to determine PC performance, specifically surface area of photocatalyst since organic pollutants degrade primarily on the surface of semiconductor. Presence of robust light absorption capacity, fast interfacial redox rate, among various nanostructures; twodimensional (2D) nanostructures tend to achieve these features more efficiently [11-14]. 2D nanomaterials also offer electron transportation channels due to reduced junctions and grain boundaries in contrast to other spherical nanocrystals. Quick transport of electrons diminish recombination rate and boost PC degradation performance. So in this line, Graphene oxide (GO) is a suitable candidate to endorse semiconductive PC efficiency [15-18].

In the last few decades, in addition to CNTs and other carbon-based nanomaterials, graphene with single atomic thick nanosheet emerged as an eye-catching candidate with a wide range of promising relevant properties including energy conversion, storage and catalytic activities [19-21]. In studies regarding water treatment and distillation; owing to large number of delocalized electrons conjugated in $\mathrm{sp}^{2}$ configuration of carbon network, graphitic carbon enriches transportation of photo-electrons and significantly enhances photo-conversion efficiency of the system. Besides, GO exhibits a high absorption ability of organic materials in an aqueous medium [22, 23]. GO and reduced graphene oxides (rGO) afford PC reaction and, owing to their narrow bandgap, are promoted as visible light active semiconductor photocatalysts. Nevertheless, room for improvement is present as photo-conversion was found to be poor caused by rapid recombination of electron-hole pairs on the surface.

Photo-conversion efficiency of photocatalysts based on $\mathrm{GO} / \mathrm{rGO}$ can be enhanced by preventing electronhole recombination. To achieve this aim, surface modifications were well developed with noble metal ions including platinum (pt), palladium (pd), silver and gold nanoparticles (NPs). The silver among most studied noble metals is considered a likely candidate for modification of graphene and its analogs for PC relevance because of its low cost, matchless optical properties, higher chemical stability and non-toxic nature. More immobility of silver nanoparticles decorated on rGO is acknowledged as enhanced performance, primarily due to increased reactive area and superior charge separation. Unique electron aggregation and transportation properties of GO through conjugated scheme drive hot electrons to reactive sites and suppress recombination [23]. Consequently, on behalf of aforesaid benefits, we aimed to synthesize different ratio of $\mathrm{Ag}$ content $(2.5,5,7.5,10)$ weight \% with rGO photocatalyst through 
hydrothermal route to examined the photocatalyst efficiency and also prepared samples would characterized through several techniques to study structural optical and electronic properties.

\section{Methods}

The current study was aimed to synthesize various concentrations of Ag into rGO nanosheets through hydrothermal route to investigate the photocatalyst efficiency.

\subsection{Materials}

Graphite (99\%) and sodium nitrate $\left(\mathrm{NaNO}_{3}\right)$ 99.9\% were procured from "Sigma-Aldrich", while sulphuric acid $\left(\mathrm{H}_{2} \mathrm{SO}_{4}, 37 \%\right)$ and phosphoric acid $\left(\mathrm{H}_{3} \mathrm{PO}_{4}\right)$ were acquired from "Analar". Silver (Ag, $\left.99.8 \%\right)$, potassium permanganate $\left(\mathrm{KMnO}_{4}, 99 \%\right)$ and hydrochloric acid $(\mathrm{HCL})$ were attained from "Merck". All chemicals were used without further purification.

\subsection{Synthesis of GO}

Modified hummers method was adopted to obtain GO. Graphite $(5 \mathrm{~g})$ and $\mathrm{NaNO}_{3}(2.5 \mathrm{~g})$ were mixed in $\mathrm{H}_{2} \mathrm{SO}_{4}(108 \mathrm{ml})$ with $12 \mathrm{ml} \mathrm{H}_{3} \mathrm{PO}_{4}$. Mixture was magnetically stirred in ice bath for 10 mins; further filtrate solution was dried in muffle furnace at $60^{\circ} \mathrm{C}$ for $2 \mathrm{~h}$ to eliminate moisture. Later, $\mathrm{KMnO}_{4}(15 \mathrm{~g})$ was added slowly at maintained temperature to below $5^{\circ} \mathrm{C}$. Suspension was transferred to ice bath for $2 \mathrm{~h}$ after vigorous stirring at $98^{\circ} \mathrm{C}$ for 60 mins while water was added continuously. Further deionized water was added until suspension volume was $400 \mathrm{ml}$ after 5 mint $\mathrm{H}_{2} \mathrm{O}_{2}(12 \mathrm{ml})$ was mixed. Finally, suspension was centrifuged and washed repeatedly with water and $\mathrm{HCL}$ product was dried at $60^{\circ} \mathrm{C}$ and $\mathrm{pH}$ of $\mathrm{GO}$ was found to be 5.7 after washing as illustrated in Fig. $1[24,25]$.

\subsection{Synthesis of $\mathrm{Ag} / \mathrm{rGO}$}

The $\mathrm{rGO}$ was extracted from $\mathrm{GO}$ by thermal reduction $\mathrm{GO}$ has been reduced by keeping it at reduction temperature $\left(300^{\circ} \mathrm{C}\right)$ a sudden change in temperature causes elimination of functional groups and oxygen atoms from carbon planes and exfoliation of GO takes place to produce rGO [26]. The rGO can be considered as chemically-derived graphene, whose structure varied from one layer to multilayers [27]. Agdoped rGO with various concentration ratios was synthesized hydrothermally, using $800 \mathrm{mg} \mathrm{GO}$ nanosheets incorporated with $(25,50,75$, and $100 \mathrm{mg}) \mathrm{Ag}$ in $80 \mathrm{ml}$ deionized water under vigorously stirring for $20 \mathrm{~min}$. The solution was then centrifuged (30 min) and subsequently transferred to $100 \mathrm{ml}$ Teflon-lined autoclave, sealed and heated up at $200^{\circ} \mathrm{C}(24 \mathrm{~h})$. The final product was dried at $\sim 200^{\circ} \mathrm{C}$ as shown in Fig. 1 [9].

\subsection{Photocatalytic activity}

Photocatalytic activity of prepared products was evaluated by degradation of synthetic methylene blue (MB) in aqueous medium as shown in Fig. 2. Dye $(5 \mathrm{mg} / 500 \mathrm{ml})$ was prepared with $10 \mathrm{mg}$ suspension of 
photocatalyst (0.025:1, 0.050:1, 0.075:1 and 0.1:1) under stirring (5 min) and exposed to dark for 30 min to achieve significant absorbance. $60 \mathrm{ml}$ of prepared solution with vigorous stirring was transferred to photo-reactor under mercury lamp (400 W and 400-700 nm) used as visible light source. After light exposure for specified time intervals $(20 \mathrm{~min})$, suspension $(3 \mathrm{ml})$ was collected to determine dye degradation. The concentration/absorbance of MB was examined with UV-Vis spectrometer; decolorization efficiency of prepared photocatalyst was evaluated as:

[Please see the supplementary files section to view the equation.]

Where $\mathrm{C}_{\mathrm{o}}$ is absorbance at $t=0$ and $C$ is absorbance at time $t$ (specific time interval) $[8,10]$.

The mechanism of photocatalytic degradation of organic molecules is elucidated as follows (Fig. 2). When photocatalyst $(\mathrm{Ag} / \mathrm{rGO})$ is irradiated with photons of energy equal to or more than bandgap energy of $\mathrm{PC}$, then electrons $\left(\mathrm{e}^{-}\right)$are excited from valence band $(\mathrm{VB})$

[Please see the supplementary files section to view the equation.]

Generated electrons through irradiation can be readily trapped by $\mathrm{O}_{2}$ absorbed molecule on surface of photocatalyst $(\mathrm{PC})$ or dissolved $\mathrm{O}_{2}$ to give superoxide radicals i.e. $\mathrm{O}_{2}{ }^{--}$

[Please see the supplementary files section to view the equation.]

Thus, $\mathrm{O}_{2}{ }^{*-}$ can react with $\mathrm{H}_{2} \mathrm{O}$ to produce hydroperoxy radical $\left(\mathrm{H}_{2} \mathrm{O}^{\circ}\right)$ and hydroxyl radical $\left(\mathrm{OH}^{\circ}\right)$, which are influential oxidizing agents that decompose organic molecules:

[Please see the supplementary files section to view the equation.]

Concurrently, photogenerated holes could be trapped by surface hydroxyl groups $\left(\mathrm{H}_{2} \mathrm{O}\right)$ on the surface of a photocatalyst to produce hydroxyl radicals $\left(\mathrm{OH}^{\circ}\right)$ :

[Please see the supplementary files section to view the equation.]

[Please see the supplementary files section to view the equation.]

Eventually, organic molecules will be oxidized to yield $\mathrm{CO}_{2}$ and $\mathrm{H}_{2} \mathrm{O}$ as follows:

[Please see the supplementary files section to view the equation.]

Temporarily, slight recombination of positive hole and electron could take place which could reduce photocatalytic activity of prepared nanocatalyst [28].

\subsection{Materials Characterization}


Crystal structure and phase-information of GO and Ag@rGO were investigated through XRD, by spectrum Bruker system (XRD, D2 Phaser, USA) equipped with monochromatized Cu K radiation of an average wavelength of $0.154 \mathrm{~nm}\left(5-80^{\circ}\right)$ using a scan rate of $0.05 / \mathrm{min}$. FTIR Perklin Elmer 3100 spectrometer with a spectral range of $4000-400 \mathrm{~cm}^{-1}$ with an increase of 32 scans and a resolution of $0.2 \mathrm{~cm}^{-1}$ was employed to detect functional groups and other molecular vibrations of prepared samples. Optical properties were recorded through UV-vis Spectrophotometer (TECAN infinite M200PRO) in the range of 200-700 nm. Surface morphology and interlayer distance of synthesized samples were observed using Field Emission Scanning Electron Microscope (FESEM), JSM-6460LV and High-Resolution Transmission Electron Microscope (HR-TEM) Philips CM30 and JEOL JEM 2100F. To confirm GO, Ag/rGO flakes and vibration modes, Raman spectra were employed on Renishaw in through Reflex confocal Raman microscope with a wavelength of $532 \mathrm{~nm}(6 \mathrm{~mW})$ laser. Photoluminescence spectra of as-prepared and doped samples were recorded through spectrofluorometer (JASCO, FP-8300).

\section{Results And Discussion}

The phase structure and crystallite size of prepared Ag inserted rGO nanosheets were examined using XRD analysis (Fig.3a). Diffractogram of GO shows intense reflection located at $\sim 10.27^{\circ}$ attributed to (001) plane with an interlayer spacing of $0.80 \mathrm{~nm}[23,29,30]$. Upon Ag doping broad peak originate at $\sim 25.4^{\circ}$, which is recognized as characteristic peak of graphene indexed as (002) plane (JCPDS No\# 040783) of hexagonal graphite, with d spacing of $0.34 \mathrm{~nm}[23,30-32]$. Peak (001) reveals the graphite powder completely oxidized into $\mathrm{GO}$ and (002) peak endorsed removal of polyhydrocarbon template in between two layers of rGO [30]. After Ag substitution, GO peak (001) shifted to a higher value to 2 at $25.4^{\circ}$ with lower $\mathrm{d}$-spacing evident to redox reaction between graphene oxide and silver ions (Ag-rGO) and d-shifting value after reduction caused by removal of oxygen-containing groups that intercalate between layers of reduced graphene oxide ( $r \mathrm{GO}$ ) as visible in XRD diffractogram [29, 32]. Average crystallite size assessed by Scherer's equation:

[Please see the supplementary files section to view the equation.] (8)

The crystallite sizes are found to be $\sim 4.85,11.3,11.53,11.6$ and $28.3 \mathrm{~nm}$ respectively. In Equation $8 \mathrm{k}=$ $0.89,=F W H M, \lambda=0.154 \mathrm{~nm}$ and $\theta=$ diffraction angle. Selected area electron diffraction (SAED) in Fig. $3(b, c)$ corresponding to XRD patterns of prepared samples, exhibits distinct ring features and indicating hexagonal phase of $\mathrm{GO}$ and $\mathrm{Ag} / \mathrm{rGO}$ manifested to well-crystallized products also ring indexing was consistent with XRD patterns.

Fourier transform infrared (FTIR) spectra of GO and Ag-doped rGO are illustrated in Fig. 3d. Observed peak $\sim 3433 \mathrm{~cm}^{-1}$ corresponds to $0-\mathrm{H}$ stretching vibration [23]. Low transmittance peaks at $1719 \mathrm{~cm}^{-}$ ${ }^{1}$ assign to $\mathrm{C}=0$ stretching vibrations caused by $\mathrm{COOH}$ groups and the band at $1627 \mathrm{~cm}^{-1}$ assigned to the aromatic C-C stretching [10, 33]. Peak at $\sim 2371 \mathrm{~cm}^{-1}$ assigned to $\mathrm{COO}$ groups [34, 35]. Transmittance peak $\left(\sim 650 \mathrm{~cm}^{-1}\right)$ is a fingerprint region of hybridized $s p^{2}$ carbon bonding allotted as $\mathrm{C}-\mathrm{H}$ bending vibration [36]. Band $\sim 1082 \mathrm{~cm}^{-1}$ corresponds to $\mathrm{C}-\mathrm{O}-\mathrm{C}$ from hydroxyl stretching vibrations, upon doping, peak 
value of functional groups on doped sample slightly changed while their shapes remain similar $[23,29$, 37].

Optical properties in terms of absorbance and bandgap analysis of Ag-rGO photocatalyst were scrutinized through Uv-vis spectrograph ranging from $200-700 \mathrm{~nm}$ as shown in Fig. 4a. The Uv-vis spectrum of GO exhibit characteristics peak around $230 \mathrm{~nm}$ owing to $\pi-\pi^{\star}$ transition of aromatic C-C bonds indicated restoring of an extensive conjugated framework of $\mathrm{sp}^{2}$ carbon atoms. Another shoulder peak observed at $300 \mathrm{~nm}$ attributed to $n-\pi^{\star}$ transitions of $C=0$ bonds $[19,23,31]$. Conversely, these two peaks became weaker in case of $\mathrm{Ag} / \mathrm{rGO}$ corresponding to $\pi-\pi^{*}$ transition of aromatic $\mathrm{C}-\mathrm{C}$ bonding found to be red-shifted at $270 \mathrm{~nm}$ that confirms reduction of $\mathrm{GO}$ and indicate no restoring of electron conjugation of graphene $[23,29]$. The absorption in visible region $(\sim 400 \mathrm{~nm})$ owing to their surface plasmonic resonance of Ag NPs, that is further evidence to as visible light active photocatalyst for removal of organic bodies $[23,29,38]$. Bandgap was calculated by Tauc equation; by plotting of vs by extrapolated of linear fits, band was calculated to be $4.10 \mathrm{eV}$ for GO and 3.98 to $3.50 \mathrm{eV}$ for $\mathrm{Ag} / \mathrm{rGO}$, bandgap gradually decreased with higher doping of Ag NPs clearly observed in Fig. 4b [39].

The morphological characteristics of GO and Ag-rGO samples elucidated through FESEM and HR-TEM showing in Fig. 5. GO Images (Fig. 5a) show few layers of microstructures with rich wrinkles and fluffy morphology resembling with thin curtain. Images of Ag@rGo (Fig. 5b-d) show partially folded and curling transparent nanosheets with small fluctuations which are essential to endure thermodynamic stability of graphene, owing to its 2D crystal structure. Nanosheets exhibit extremely clean, silky and wavy structures and this feature may be important to avoid aggregation of rGO sheets and maintain surface to facilitate attachment of Ag NPs on graphene sheets that can be visualized in HR-TEM images [37]. The corresponding HR-TEM images (Fig. $6 \mathrm{a}_{1}-\mathrm{d}_{1}$ ), GO exhibits lamellar and sheet-like structure with clean surface area (Fig. 6a $\mathrm{a}_{1}$ ), in Ag-rGO sample (Fig. 5 $\mathrm{b}_{1}$ ) few stacking folds owing to their distortions from high fraction of $s p^{3} \mathrm{C}-\mathrm{O}$ bonds [29]. With increasing concentration of Ag NPs (Fig. 6c $\mathrm{c}_{1}, \mathrm{~d}_{1}$ ) images revealed a well-dispersed and homogeneous scattering of spherical shaped Ag NPs on the surface of rGO sheets with average particle size of $10-12 \mathrm{~nm}[23,29]$. In Fig. $6 \mathrm{~d}_{1}$ with a higher concentration $(10 \%)$ of Ag, aggregation of particles increased which is evident to doped species.

The extremely high resolution images up to 5 and $10 \mathrm{~nm}$ d-spacing of $\mathrm{Ag} / \mathrm{rGO}$ samples can be clearly observed in Fig. 6 (a-d). Circled areas indicate the presence of Ag NPs with lattice spacing of Ag nanocrystal being approximately $0.235 \mathrm{~nm}[23,29]$.

Photoluminescence (PL) analysis was conducted to investigate lifetime, transfer and trapping of electronhole pair and study of interaction between graphene nanostructures, its influence on photocatalytic response illustrated in Fig. 7a [40,41]. Graphite exhibits no luminescence properties due to zero bandgap. Nevertheless, upon decrease in size up to nano-scale, bandgap becomes wide caused by quantum confinement effect. In nanosheets of $\mathrm{GO}$ and $\mathrm{rGO}$, oxides groups and carbon vacancies altered the graphene to form any carbon nano-cluster that demonstrates semiconductive behavior and luminescence phenomenon which can be influenced by size or fraction of chains and clusters [41, 42]. In PL spectra, 
luminescence peaks were located at $\sim 330,565$ and $608 \mathrm{~nm}$ which is ascribed to electron-hole pair recombination in local state of $\mathrm{sp}^{2}$ carbon clusters incorporated with $\mathrm{sp}^{3}$ matrix. Therefore, $\mathrm{rGO}$ luminescence is due to disappeared oxygen functional groups that facilitate percolation of pathways between $\mathrm{sp}^{2}$ clusters [41]. Significant peak at $\sim 565 \mathrm{~nm}$ sharply decreased in case of rGO with reduction of $\mathrm{GO}$ oxide functional groups that are decreased and $\mathrm{sp}^{2}$ carbon cluster are expanded simultaneously [42].

Raman spectroscopy was deployed to probe electronic and structural properties of control sample and Ag@rGO for distinguishing ordered and disordered carbon structures as demonstrated in Fig. 7(b-c). In case of GO, two bands are located at $\sim 1340$ and $\sim 1590 \mathrm{~cm}^{-1}$ assigned as $\mathrm{D}$ and $\mathrm{G}$ band, respectively. The $D$ band is assigned to breathing mode of k-point phonons with $\mathrm{A}_{1 \mathrm{~g}}$ symmetry and band from $\mathrm{sp}^{3}$ carbon atoms; $\mathrm{G}$ band suggests a characteristic peak of $\mathrm{sp}^{2}$ hybrid structure which reveals symmetry and crystallizability of carbon and introduces $E_{2 g}$ phonon scattering of carbon atoms [32, 33, 37]. Moreover, $D$ band is evident to surface defects and structural imperfections arise with attached hydroxyl and epoxide functions groups with carbon basal planes [37]. The $\mathrm{G}$ band is only Raman mode in graphene originating from a conventional first-order Raman scattering process and corresponds to in-plane zone center, doubly degenerate phonon mode (transverse (TO) and longitudinal (LO) optical) with $\mathrm{E}_{2 \mathrm{~g}}$ symmetry [43]. In case of Ag-rGO Raman spectrum observed at $1338 \mathrm{~cm}^{-1}$ (D band), $1583 \mathrm{~cm}^{-1}$ (G band) and $2682 \mathrm{~cm}^{-1}$ (2D band) there is an additional peak centered at $2900 \mathrm{~cm}^{-1}$ ( $D+G$ band) that represents disorder due to combination scattering in Fig. $7(b-c)[31,36,43-46]$. The $D$ and $2 D$ modes originate from second-order double resonant process between non-equivalent $\mathrm{K}$ points in Brillouin zone (BZ) of graphene, as 2D band indicates second order of $D$ band which alludes to overtone of $D$ band with its existence owing two phone lattice vibrational processes; nevertheless, it is not associated with defects like $D$ band in Fig. 7c [36, 42]. The variations in relative intensities of $G$ and $D$ band in Raman spectra of GO during reduction are usually designated to a change in electronic conjugation state. This change suggests an increase in number of $s p^{2}$ atomic domains following reduction of $\mathrm{GO}$ [47]. The intensity ratio of $\mathrm{D}$ to $\mathrm{G}$ band defines disorder degree in graphite layers; $I_{D} / I_{G}=0.87$ for doped free sample $(G O), I_{D} / I_{G}=1.15$ for Ag-doped samples and increase in ratio indicates decrease in average size of $\mathrm{sp}^{2}$ carbon domains after synthesis of Ag@rGo, while intensity ratio between $2 \mathrm{D}$ and $\mathrm{G}$ band $\left(\mathrm{I}_{2 \mathrm{D}} / \mathrm{I}_{\mathrm{G}}\right)$ which is 1.69 , have been used to probe electrons concentration in rGO [31-32, 36, 48].

Ag nanoparticles, when doped in semiconductor material, generate a contact potential difference due to their different work functions. This potential difference is called the Schottky barrier. As shown in Fig. 8, the band bending when a contact is formed after reaching equilibrium is dependent on the relative energies of the work functions of metal $\left(\phi_{M}\right)$ and the semiconducting $\left(\phi_{B}\right)$ components. This phenomenon can greatly enhance the charge separation efficiency, once it can induce the directional migration of photogenerated electrons from the semiconductor to the metal. In other words, it can lead to the generation of effective electron trapping site to suppress the electron-hole recombination [49]. 
The photocatalytic activity of $\mathrm{GO}$ and $\mathrm{Ag} / \mathrm{rGO}$ nanosheets occurred due to their high surface area, and low band-gap energy. Thus $\mathrm{Ag} / \mathrm{rGO}$ exhibits substantial improvement in photo-degradation of MB and the dye degrades completely (Fig. 9b) in $120 \mathrm{~min}$. The pseudo-first-order equation can be employed to elaborate on photocatalytic efficiency (Fig. 8a) of GO and Ag/rGO samples explicitly, using following expression.

[Please see the supplementary files section to view the equation.]

where $C_{o}$ is initial concentration of dye and $C_{t}$ is concentration at time, $k$ is apparent rate constant of degradation process that shows in absorbance plot (Fig. 9a) i.e., value of $\mathrm{k}$ for $\mathrm{GO}$ is about $0.1300 \mathrm{~min}^{-1}$ and $\mathrm{k}$ extraordinarily increases in case of $\mathrm{Ag} / \mathrm{rGO}\left(0.1300 \mathrm{~min}^{-1}\right.$ to $\left.0.7459 \mathrm{~min}^{-1}\right)$. Fig. $9 \mathrm{c}$ reveals compression of $\%$ degradation with time, $\mathrm{GO}$ shows $65 \%$ efficiency and a gradual increase with doping concentration. $\mathrm{Ag} / \mathrm{rGO}(0.10: 1)$ shows maximum \%degradation up to $100 \%$ which is likely due to synergetic effects of Ag NPs $[50,51]$. Finally, on the basis of these findings in the present study, it can be suggested that $\mathrm{Ag} / \mathrm{rGO}$ is an excellent product that can be used for purification of water from organic dyes.

\section{Conclusion}

GO was successfully obtained through Modified hummers method and rGO was synthesized from thermal treatment during insertion of $\mathrm{Ag}(2.5,5,7.5$ and $10 \mathrm{wt} . \%)$ via Hydrothermal route. According to XRD pattern, peak shift and decrease in d-spacing $(0.34$ to $.023 \mathrm{~nm})$ point toward redox reaction of $\mathrm{GO}$ upon Ag-doping with hexagonal crystal structure; Average crystallite size increase (4.85 to $15.6 \mathrm{~nm}$ ) with substitution of Ag is observed. FTIR spectra confirmed transmittance peak around $650 \mathrm{~cm}^{-1}$ which is a fingerprint region of hybridized $s p^{2}$ carbon bonding allotted as $\mathrm{C}-\mathrm{H}$ bending vibration and reveals information about other attached functional groups. The characteristic peak attributed to $\pi-\pi^{\star}$ and $n-\pi^{\star}$ bonding and redshift in peaks. It endorses the presence of Ag as elucidated with UV-vis spectroscopy, an obvious decrease in bandgap energy (4.10 to $3.50 \mathrm{eV}$ ) with increased doping ratio that was calculated with the help of Tauc equation. Morphological features show stacking layers of $\mathrm{GO}$ and $\mathrm{Ag} / \mathrm{rGO}$ with a lattice spacing of $\sim 0.235 \mathrm{~nm}$, spherical shape and size $(10-12 \mathrm{~nm})$ of Ag NPs visualized through HR-TEM. The carbon atoms of local state $\mathrm{sp}^{2}$ clusters incorporated with $\mathrm{sp}^{3}$ matrix, significant peak decrease in case of $\mathrm{rGO}$ and expanded $\mathrm{sp}^{2}$ carbon cluster upon doping was confirmed with PL spectra. $\mathrm{A}_{1 \mathrm{~g}}$ symmetry in $\mathrm{sp}^{3}$ carbons atoms at $\mathrm{D}$ band, $\mathrm{sp}^{2}$ hybrid structure that reveals symmetry and crystallizability of carbon and introduces $\mathrm{E} 2 \mathrm{~g}$ phonon scattering of a carbon atom and surface defects were calculated through Raman spectra. Photocatalytic activity responds to $\mathrm{Ag} / \mathrm{rGO}(0.10: 1)$ and degrades $100 \%$ of $\mathrm{MB}$ concentration. These findings suggest that prepared nanocatalyst shows no hazards behavior in water treatment and is an excellent nanocatalyst for elimination of organic pollutants from wastewater.

\section{Declarations}


Availability of Data and Materials

All data are fully available without restriction.

\section{Competing Interests}

The authors declare that they have no competing interests.

\section{Funding}

Authors are grateful to higher education commission (HEC), Pakistan for financial support through start research group (SRGP) project number 21-1669.

\section{AUTHORS' CONTRIBUTIONS}

$\mathrm{MI}$ and AR performed the whole experiments and wrote the manuscript. MI provided the novel idea to carry out the experiment. M Imran participated in the data analysis of the results and discussion portion. AUH reviewed the manuscript and corrected the English and facilitated in FESEM and HRTEM. All authors read and approved the final manuscript.

ACKNOWLEDGMENT: Support provided by the Research Institute, King Fahd University of Petroleum \& Minerals in transmission electron microscopy is appreciated.

\section{ABBREVIATIONS}

Ultra-violet visible spectroscopy (UV-Vis), X-ray diffraction (XRD), Fourier transform infrared spectroscopy (FTIR), Energy dispersive x-ray spectroscopy (EDS), Scanning electron microscopy (SEM) and transmission electron microscopy (TEM). JCPDS: Joint committee on powder diffraction standards.

\section{Author details}

aSolar Cell Applications Research Lab, Department of Physics, Government College University Lahore, 54000, Punjab, Paksitan

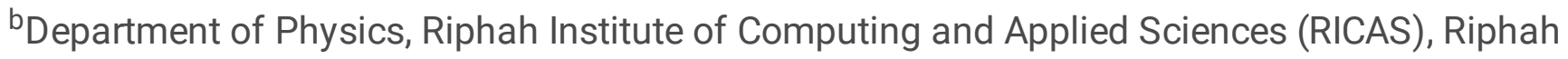
International University, 14 Ali Road, Lahore, Pakistan

'State key Laboratory of Chemical Resource Engineering, Beijing Advanced Innovation Centre for Soft Matter Science and Engineering, Beijing Engineering Center for Hierarchical Catalysts, Beijing University of Chemical Technology, Beijing 100029, China

${ }^{d}$ Center for Engineering Research, Research Institute, King Fahd University of Petroleum \& Minerals, Dhahran 31261, Saudi Arabia. 


\section{References}

[1] Z. Wang, A. Wu, L. Colombi Ciacchi, and G. Wei, "Recent Advances in Nanoporous Membranes for Water Purification," Nanomaterials, vol. 8, no. 2, p. 65, Jan. 2018, doi: 10.3390/nano8020065.

[2] S. Dervin, D. D. Dionysiou, and S. C. Pillai, "2D nanostructures for water purification: graphene and beyond," Nanoscale, vol. 8, no. 33, pp. 15115-15131, 2016, doi: 10.1039/C6NR04508A.

[3] J. Moma and J. Baloyi, "Modified Titanium Dioxide for Photocatalytic Applications," in Photocatalysts - Applications and Attributes, S. Bahadar Khan and K. Akhtar, Eds. IntechOpen, 2019.

[4] M. Sharma, K. Behl, S. Nigam, and M. Joshi, "TiO2-GO nanocomposite for photocatalysis and environmental applications: A green synthesis approach," Vacuum, vol. 156, pp. 434-439, Oct. 2018, doi: 10.1016/j.vacuum.2018.08.009.

[5] M. Junaid et al., "The study of Fe-doped CdS nanoparticle-assisted photocatalytic degradation of organic dye in wastewater," Appl Nanosci, vol. 9, no. 8, pp. 1593-1602, Nov. 2019, doi: 10.1007/s13204018-0933-3.

[6] S. O.-B. Oppong, F. Opoku, W. W. Anku, E. M. Kiarii, and P. P. Govender, “Experimental and Computational Design of Highly Active Ce-ZrO2-GO Photocatalyst for Eosin Yellow Dye Degradation: The Role of Interface and Ce3+ Ion," Catal Lett, vol. 149, no. 6, pp. 1633-1650, Jun. 2019, doi: 10.1007/s10562-019-02729-3.

[7] K. R. Kunduru, "2 - Nanotechnology for water purification: applications of nanotechnology methods in wastewater treatment," p. 42.

[8] A. Rafiq et al., "Photocatalytic and catalytic degradation of organic dye by uncapped and capped ZnS quantum dots," Mater. Res. Express, vol. 6, no. 5, p. 055801, Feb. 2019, doi: 10.1088/2053-1591/aaff8e.

[9] F. Khurshid, M. Jeyavelan, M. S. L. Hudson, and S. Nagarajan, “Ag-doped ZnO nanorods embedded reduced graphene oxide nanocomposite for photo-electrochemical applications," $R$. Soc. open sci., vol. 6, no. 2, p. 181764, Feb. 2019, doi: 10.1098/rsos.181764.

[10] M. Aqeel et al., "TiO 2 @ RGO (reduced graphene oxide) doped nanoparticles demonstrated improved photocatalytic activity," Mater. Res. Express, vol. 6, no. 8, p. 086215, May 2019, doi: 10.1088/20531591/ab244a.

[11] M. Zhang et al., "3D hierarchical CoWO4/Co3O4 nanowire arrays for asymmetric supercapacitors with high energy density," Chemical Engineering Journal, vol. 347, pp. 291-300, Sep. 2018, doi: 10.1016/j.cej.2018.04.113.

[12] Y. Zhao, H. Fan, K. Fu, L. Ma, M. Li, and J. Fang, "Intrinsic electric field assisted polymeric graphitic carbon nitride coupled with Bi4Ti3012/Bi2Ti2O7 heterostructure nanofibers toward enhanced 
photocatalytic hydrogen evolution," International Journal of Hydrogen Energy, vol. 41, no. 38, pp. 1691316926, Oct. 2016, doi: 10.1016/j.jhydene.2016.07.162.

[13] X. Zou, H. Fan, Y. Tian, M. Zhang, and X. Yan, "Microwave-assisted hydrothermal synthesis of $\mathrm{Cu} / \mathrm{Cu}$ ${ }_{2} \mathrm{O}$ hollow spheres with enhanced photocatalytic and gas sensing activities at room temperature," Dalton Trans., vol. 44, no. 17, pp. 7811-7821, 2015, doi: 10.1039/C4DT03417A.

[14] C. Wang, H. Fan, X. Ren, Y. Wen, and W. Wang, "Highly dispersed PtO nanodots as efficient co-catalyst for photocatalytic hydrogen evolution," Applied Surface Science, vol. 462, pp. 423-431, Dec. 2018, doi: 10.1016/j.apsusc.2018.08.126.

[15] A. Raza et al., "Enhanced industrial dye degradation using Co doped in chemically exfoliated MoS2 nanosheets," App/ Nanosci, Dec. 2019, doi: 10.1007/s13204-019-01239-3.

[16] S. Kumar, C. Terashima, A. Fujishima, V. Krishnan, and S. Pitchaimuthu, "Photocatalytic Degradation of Organic Pollutants in Water Using Graphene Oxide Composite," in A New Generation Material Graphene: Applications in Water Technology, Mu. Naushad, Ed. Cham: Springer International Publishing, 2019, pp. 413-438.

[17] J. Di, J. Xia, H. Li, and Z. Liu, "Freestanding atomically-thin two-dimensional materials beyond graphene meeting photocatalysis: Opportunities and challenges," Nano Energy, vol. 35, pp. 79-91, May 2017, doi: 10.1016/j.nanoen.2017.03.030.

[18] Y. Li, C. Gao, R. Long, and Y. Xiong, "Photocatalyst design based on two-dimensional materials," Materials Today Chemistry, vol. 11, pp. 197-216, Mar. 2019, doi: 10.1016/j.mtchem.2018.11.002.

[19] M. Mitra et al., "Polyaniline/Reduced Graphene Oxide Composite-Enhanced Visible-Light-Driven Photocatalytic Activity for the Degradation of Organic Dyes," ACS Omega, vol. 4, no. 1, pp. 1623-1635, Jan. 2019, doi: 10.1021/acsomega.8b02941.

[20] P. Raizada, A. Sudhaik, and P. Singh, "Photocatalytic water decontamination using graphene and ZnO coupled photocatalysts: A review," Materials Science for Energy Technologies, vol. 2, no. 3, pp. 509-525, Dec. 2019, doi: 10.1016/j.mset.2019.04.007.

[21] W. Vallejo, A. Rueda, C. Díaz-Uribe, C. Grande, and P. Quintana, "Photocatalytic activity of graphene oxide- $\mathrm{TiO}_{2}$ thin films sensitized by natural dyes extracted from Bactris guineensis," R. Soc. open sci., vol. 6, no. 3, p. 181824, Mar. 2019, doi: 10.1098/rsos.181824.

[22] E. Dadvar, R. R. Kalantary, H. Ahmad Panahi, and M. Peyravi, "Efficiency of Polymeric Membrane Graphene Oxide-TiO 2 for Removal of Azo Dye," Journal of Chemistry, vol. 2017, pp. 1-13, 2017, doi: $10.1155 / 2017 / 6217987$. 
[23] A. Kumar, A. M. Sadanandhan, and S. L. Jain, "Silver doped reduced graphene oxide as a promising plasmonic photocatalyst for oxidative coupling of benzylamines under visible light irradiation," New J. Chem., vol. 43, no. 23, pp. 9116-9122, 2019, doi: 10.1039/C9NJ00852G.

[24] J. Song, X. Wang, and C.-T. Chang, "Preparation and Characterization of Graphene Oxide," Journal of Nanomaterials, vol. 2014, pp. 1-6, 2014, doi: 10.1155/2014/276143.

[25] S. N. Alam, N. Sharma, and L. Kumar, "Synthesis of Graphene Oxide (GO) by Modified Hummers Method and Its Thermal Reduction to Obtain Reduced Graphene Oxide (rGO)*," Graphene, vol. 06, no. 01, pp. 1-18, 2017, doi: 10.4236/graphene.2017.61001.

[26] I. Sengupta, S. Chakraborty, M. Talukdar, S. K. Pal, and S. Chakraborty, "Thermal reduction of graphene oxide: How temperature influences purity," J. Mater. Res., vol. 33, no. 23, pp. 4113-4122, Dec. 2018, doi: 10.1557/jmr.2018.338.

[27] G. T. T. Le, J. Manyam, P. Opaprakasit, N. Chanlek, N. Grisdanurak, and P. Sreearunothai, "Divergent mechanisms for thermal reduction of graphene oxide and their highly different ion affinities," Diamond and Related Materials, vol. 89, pp. 246-256, Oct. 2018, doi: 10.1016/j.diamond.2018.09.006.

[28] A. Gnanaprakasam, V. M. Sivakumar, and M. Thirumarimurugan, "Influencing Parameters in the Photocatalytic Degradation of Organic Effluent via Nanometal Oxide Catalyst: A Review," Indian Journal of Materials Science, vol. 2015, pp. 1-16, 2015, doi: 10.1155/2015/601827.

[29] B. Fan et al., "Facile One-Pot Preparation of Silver/Reduced Graphene Oxide Nanocomposite for Cancer Photodynamic and Photothermal Therapy," j nanosci nanotechnol, vol. 16, no. 7, pp. 7049-7054, Jul. 2016, doi: 10.1166/jnn.2016.11327.

[30] S. Some et al., "High-Quality Reduced Graphene Oxide by a Dual-Function Chemical Reduction and Healing Process," Sci Rep, vol. 3, no. 1, p. 1929, Dec. 2013, doi: 10.1038/srep01929.

[31] A. Shaikh, S. Parida, and S. Böhm, "One step eco-friendly synthesis of Ag-reduced graphene oxide nanocomposite by phytoreduction for sensitive nitrite determination," RSC Adv., vol. 6, no. 102, pp. 100383-100391, 2016, doi: 10.1039/C6RA23655C.

[32] Walton, Ian M., Jordan M. Cox, Cassidy A. Benson, Dinesh Dan G. Patel, Yu-Sheng Chen, and Jason B. Benedict. "The role of atropisomers on the photo-reactivity and fatigue of diarylethene-based metalorganic frameworks." New Journal of Chemistry 40, no. 1 (2016): 101-106.

[33] J. A. Luceño-Sánchez, G. Maties, C. Gonzalez-Arellano, and A. M. Díez-Pascual, "Synthesis and Characterization of Graphene Oxide Derivatives via Functionalization Reaction with Hexamethylene Diisocyanate," Proceedings, vol. 3, no. 1, p. 8, Aug. 2018, doi: 10.3390/IOCN_2018-1-05485.

[34] Pusty, M., Rana, A. K., Kumar, Y., Sathe, V., Sen, S., \& Shirage, P. (2016). Synthesis of Partially Reduced Graphene Oxide/Silver Nanocomposite and Its Inhibitive Action on Pathogenic Fungi Grown 
Under Ambient Conditions. ChemistrySelect, 1(14), 4235-4245. doi:10.1002/slct.201600783

[35] Pusty, M., Rana, A. K., Kumar, Y., Sathe, V., Sen, S., \& Shirage, P. (2016). Synthesis of Partially Reduced Graphene Oxide/Silver Nanocomposite and Its Inhibitive Action on Pathogenic Fungi Grown Under Ambient Conditions. ChemistrySelect, 1(14), 4235-4245. doi:10.1002/slct.201600783.

[36] N. P. D. Ngidi, M. A. Ollengo, and V. O. Nyamori, "Effect of Doping Temperatures and Nitrogen Precursors on the Physicochemical, Optical, and Electrical Conductivity Properties of Nitrogen-Doped Reduced Graphene Oxide," Materials, vol. 12, no. 20, p. 3376, Oct. 2019, doi: 10.3390/ma12203376.

[37] S. Gurunathan et al., "Reduced graphene oxide\&ndash;silver nanoparticle nanocomposite: a potential anticancer nanotherapy," IJN, p. 6257, Oct. 2015, doi: 10.2147/IJN.S92449.

[38] Y. Haldorai, B.-K. Kim, Y.-L. Jo, and J.-J. Shim, “Ag@graphene oxide nanocomposite as an efficient visible-light plasmonic photocatalyst for the degradation of organic pollutants: A facile green synthetic approach," Materials Chemistry and Physics, vol. 143, no. 3, pp. 1452-1461, Feb. 2014, doi:

10.1016/j.matchemphys.2013.11.065.

[39] J. Mangalam, M. Kumar, M. Sharma, and M. Joshi, "High adsorptivity and visible light assisted photocatalytic activity of silver/reduced graphene oxide $(\mathrm{Ag} / \mathrm{rGO})$ nanocomposite for wastewater treatment," Nano-Structures \& Nano-Objects, vol. 17, pp. 58-66, Feb. 2019, doi: 10.1016/j.nanoso.2018.11.003.

[40] M. Aleksandrzak, W. Kukulka, and E. Mijowska, "Graphitic carbon nitride/graphene oxide/reduced graphene oxide nanocomposites for photoluminescence and photocatalysis," Applied Surface Science, vol. 398, pp. 56-62, Mar. 2017, doi: 10.1016/j.apsusc.2016.12.023.

[41] N. Bano, I. Hussain, A. M. EL-Naggar, and A. A. Albassam, "Reduced graphene oxide nanocomposites for optoelectronics applications," Appl. Phys. A, vol. 125, no. 3, p. 215, Mar. 2019, doi: 10.1007/s00339019-2518-8.

[42] J. J. Ding, H. X. Chen, D. Q. Feng, and H. W. Fu, "Investigation on photoluminescence emission of (reduced) graphene oxide paper," IOP Conf. Ser.: Mater. Sci. Eng., vol. 292, p. 012097, Jan. 2018, doi: $10.1088 / 1757-899 X / 292 / 1 / 012097$.

[43] O. Frank et al., "Raman 2D-Band Splitting in Graphene: Theory and Experiment," ACS Nano, vol. 5, no. 3, pp. 2231-2239, Mar. 2011, doi: 10.1021/nn103493g.

[44] S. Kr. Tiwari, G. Hatui, R. Oraon, A. De Adhikari, and G. C. Nayak, "Mixing sequence driven controlled dispersion of graphene oxide in PC/PMMA blend nanocomposite and its effect on thermo-mechanical properties," Current Applied Physics, vol. 17, no. 9, pp. 1158-1168, Sep. 2017, doi:

10.1016/j.cap.2017.05.007. 
[45] A. Kaniyoor and S. Ramaprabhu, "A Raman spectroscopic investigation of graphite oxide derived graphene," AIP Advances, vol. 2, no. 3, p. 032183, Sep. 2012, doi: 10.1063/1.4756995.

[46] S. Jaworski et al., "Graphene Oxide-Based Nanocomposites Decorated with Silver Nanoparticles as an Antibacterial Agent," Nanoscale Res Lett, vol. 13, no. 1, p. 116, Dec. 2018, doi: 10.1186/s11671-0182533-2.

[47] S. Gurunathan, J. W. Han, J. H. Park, V. Eppakayala, and J.-H. Kim, “Ginkgo biloba: a natural reducing agent for the synthesis of cytocompatible graphene," IJN, p. 363, Jan. 2014, doi: 10.2147/IJN.S53538.

[48] G. Yasin et al., "Exploring the Nickel-Graphene Nanocomposite Coatings for Superior Corrosion Resistance: Manipulating the Effect of Deposition Current Density on its Morphology, Mechanical Properties, and Erosion-Corrosion Performance," Adv. Eng. Mater., vol. 20, no. 7, p. 1701166, Jul. 2018, doi: 10.1002/adem.201701166.

[49] I. Teixeira, J. Quiroz, M. Homsi, and P. Camargo, "An Overview of the Photocatalytic H2 Evolution by Semiconductor-Based Materials for Nonspecialists," J. Braz. Chem. Soc., 2020, doi: 10.21577/01035053.20190255.

[50] G. Zeng et al., "Rapid synthesis of $\mathrm{MoS}_{2}$-PDA-Ag nanocomposites as heterogeneous catalysts and antimicrobial agents via microwave irradiation," Applied Surface Science, vol. 459, pp. 588-595, Nov. 2018, doi: 10.1016/j.apsusc.2018.07.144.

[51] A. Arshad et al., "Solar light triggered catalytic performance of graphene-CuO nanocomposite for waste water treatment," Ceramics International, vol. 43, no. 14, pp. 10654-10660, Oct. 2017, doi: 10.1016/j.ceramint.2017.03.165.

\section{Figures}




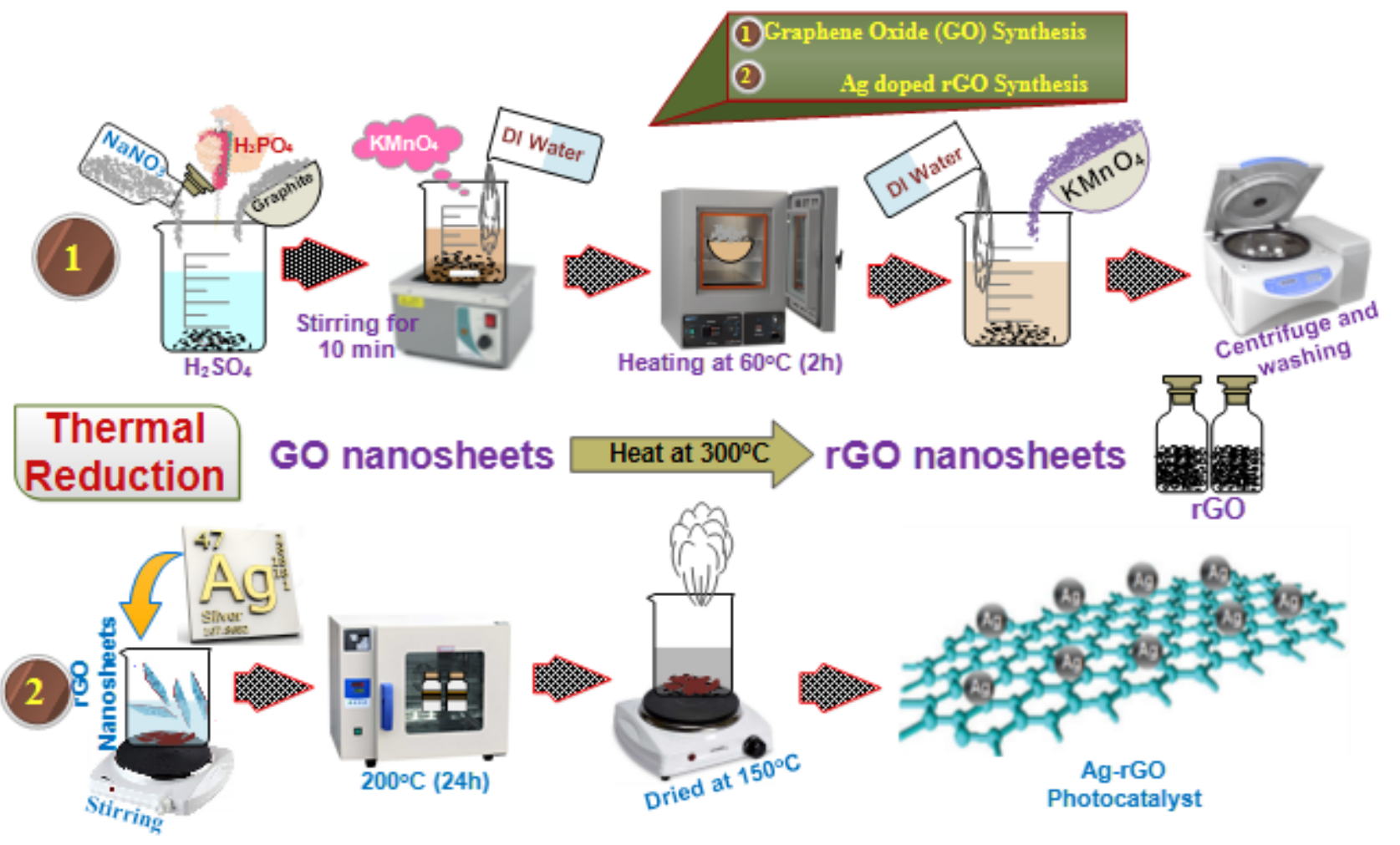

Figure 1

Synthesis process for $\mathrm{GO}$ and $\mathrm{Ag}$ doped $\mathrm{rGO}$

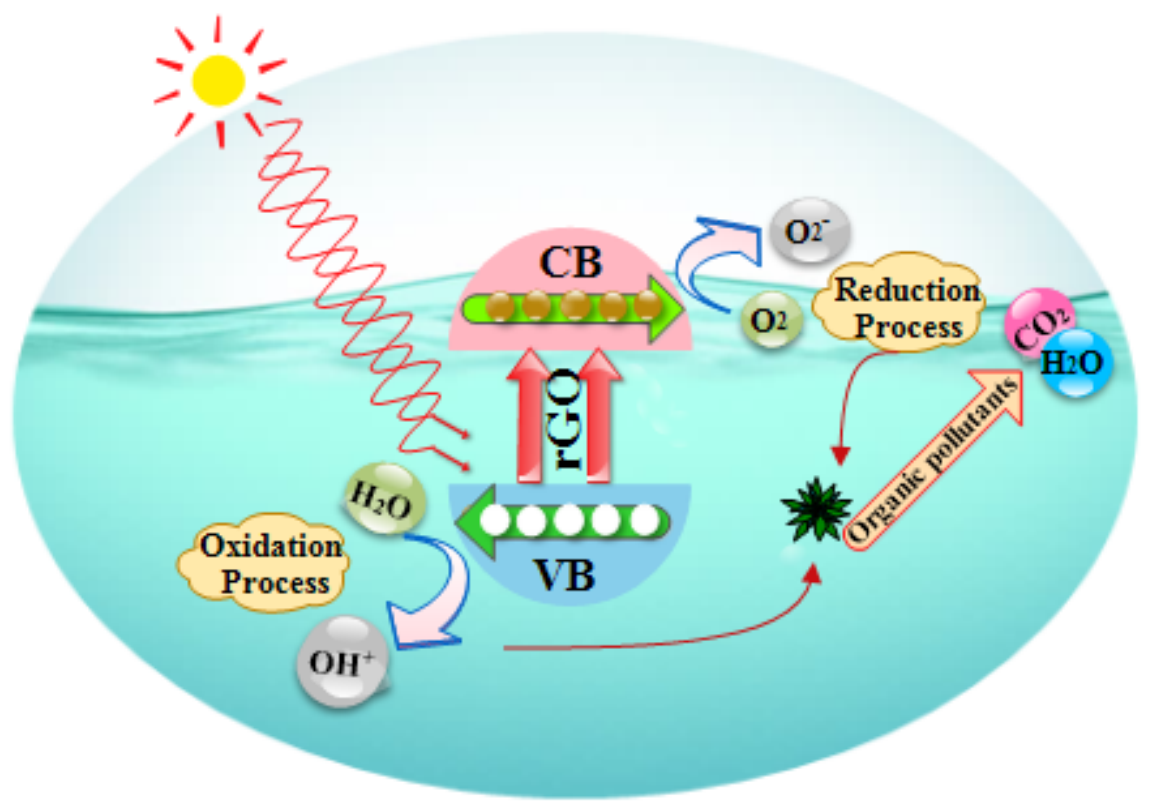

Figure 2

Photocatalytic mechanism for dye degradation in the presence of $\mathrm{Ag} / \mathrm{rGO}$ 

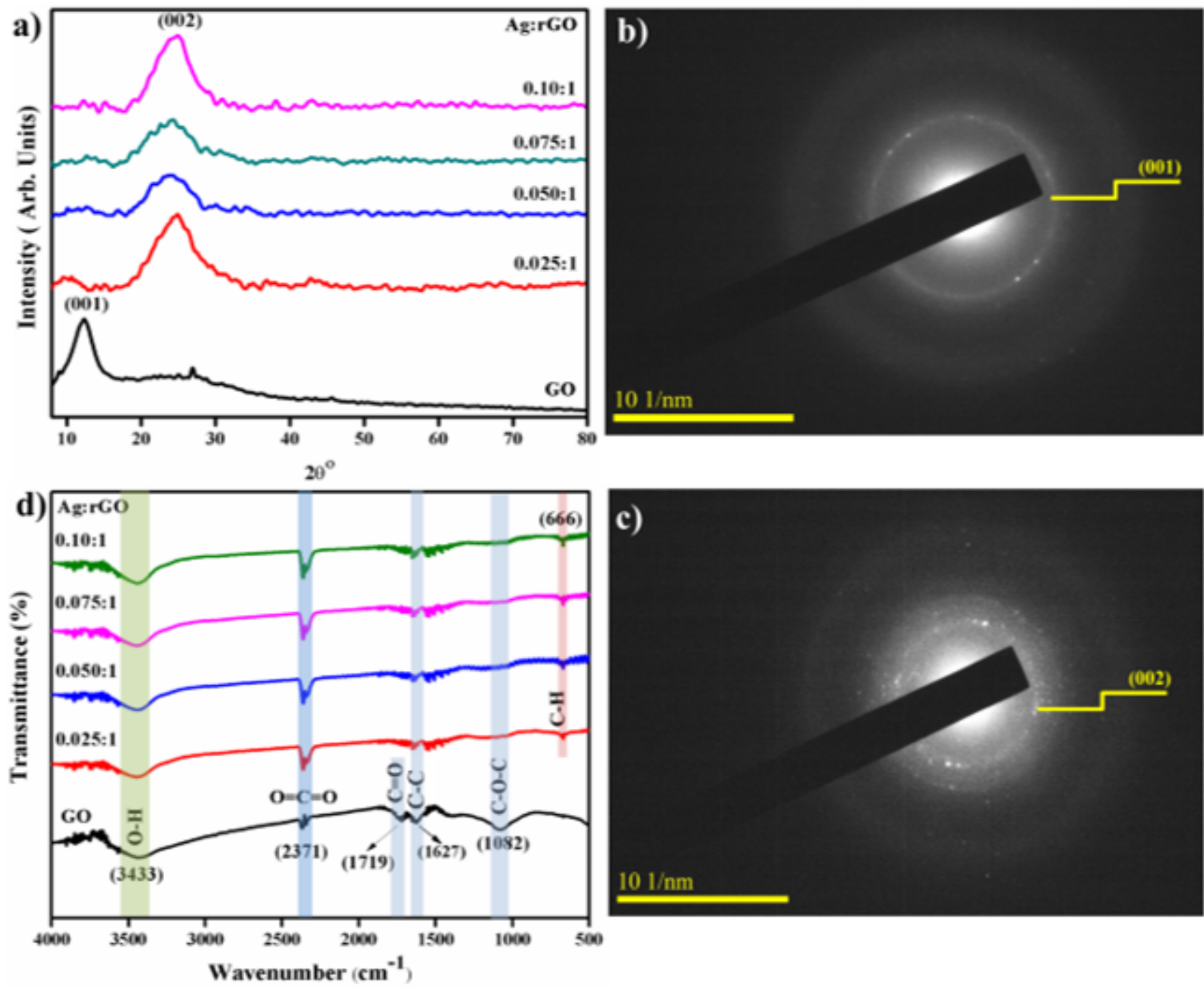

Figure 3

(a) XRD pattern (b-c) SAED rings of as-prepared and Ag-doped RGO (b) 0:1 (c) 0.010:1 (d) FTIR Spectra
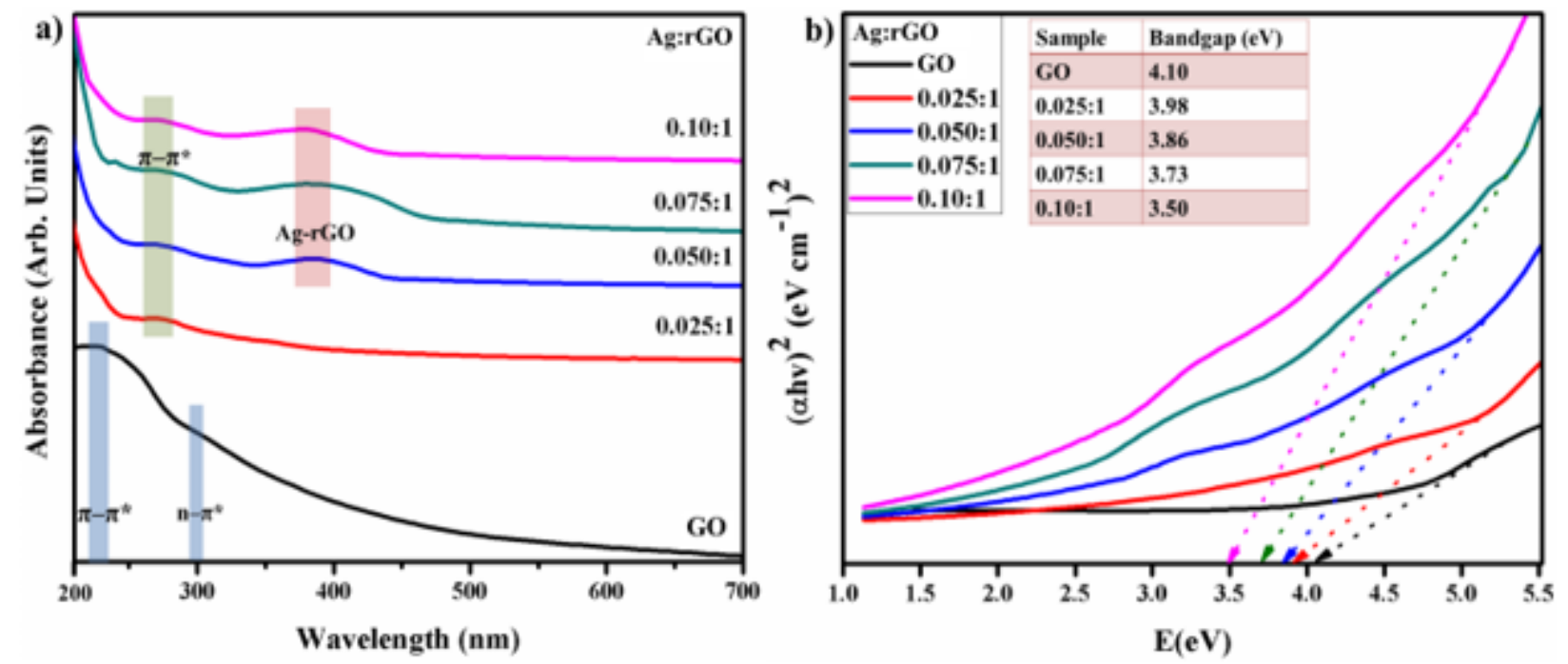

Figure 4 
(a) UV-vis. spectra of GO and Ag-rGO (b) Bandgap comparison
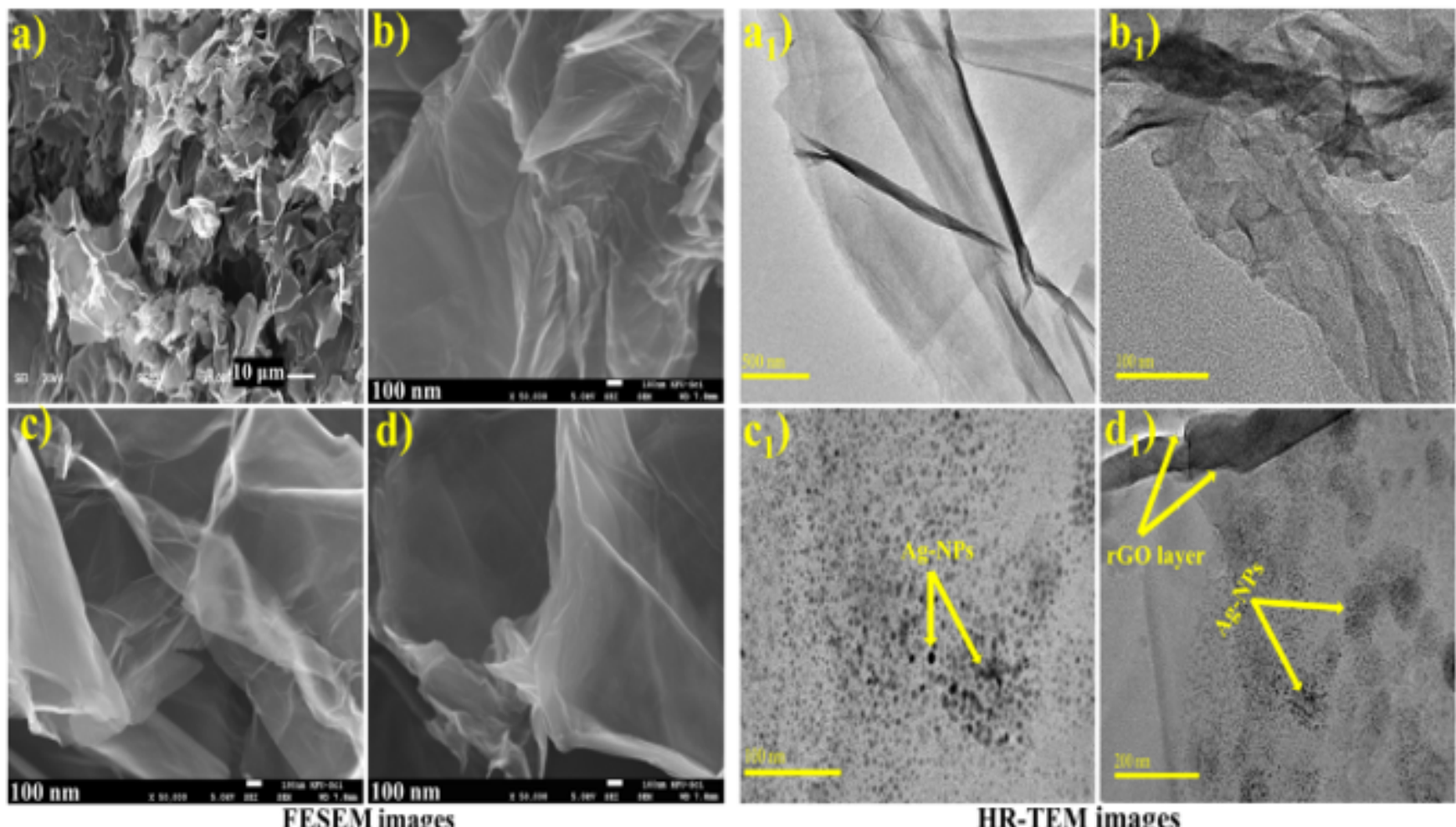

HR-TEM images

\section{Figure 5}

(a-d, a1-d1) FESEM and HR-TEM images of GO and Ag/rGO (a, a1) GO (b, b1) 0.050:1 (c, c1) 0.075:1 and (d, d1) 0.10:1 

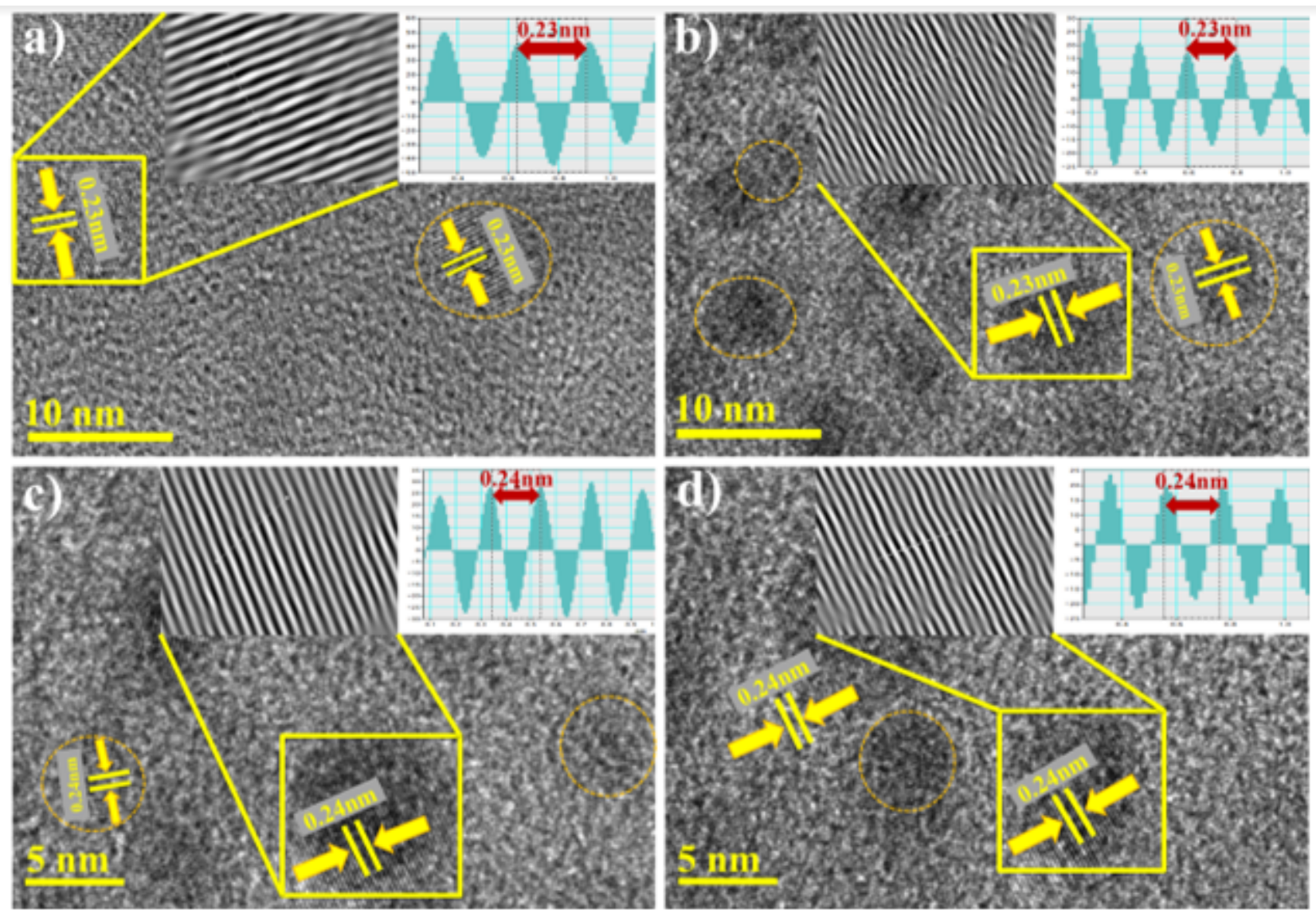

\section{Figure 6}

(a-d) d-spacing from HR-TEM images of Ag-rGO (a) 0.025:1 (b) 0.050:1 (c) 0.075:1 (d) 0.10:1 

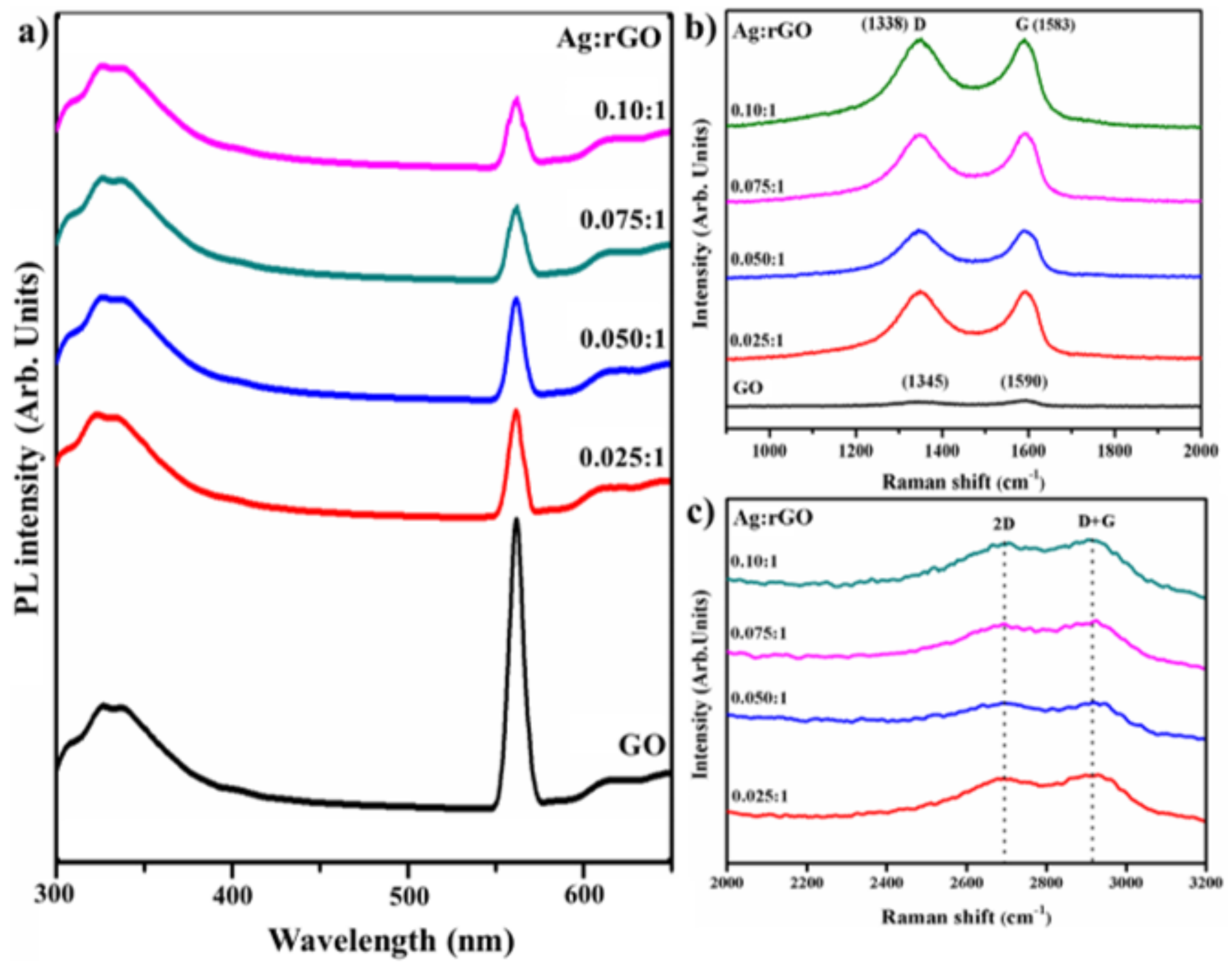

Figure 7

(a) PL spectra (b) Raman spectra of prepared samples (c) Zoom area of Raman spectra 


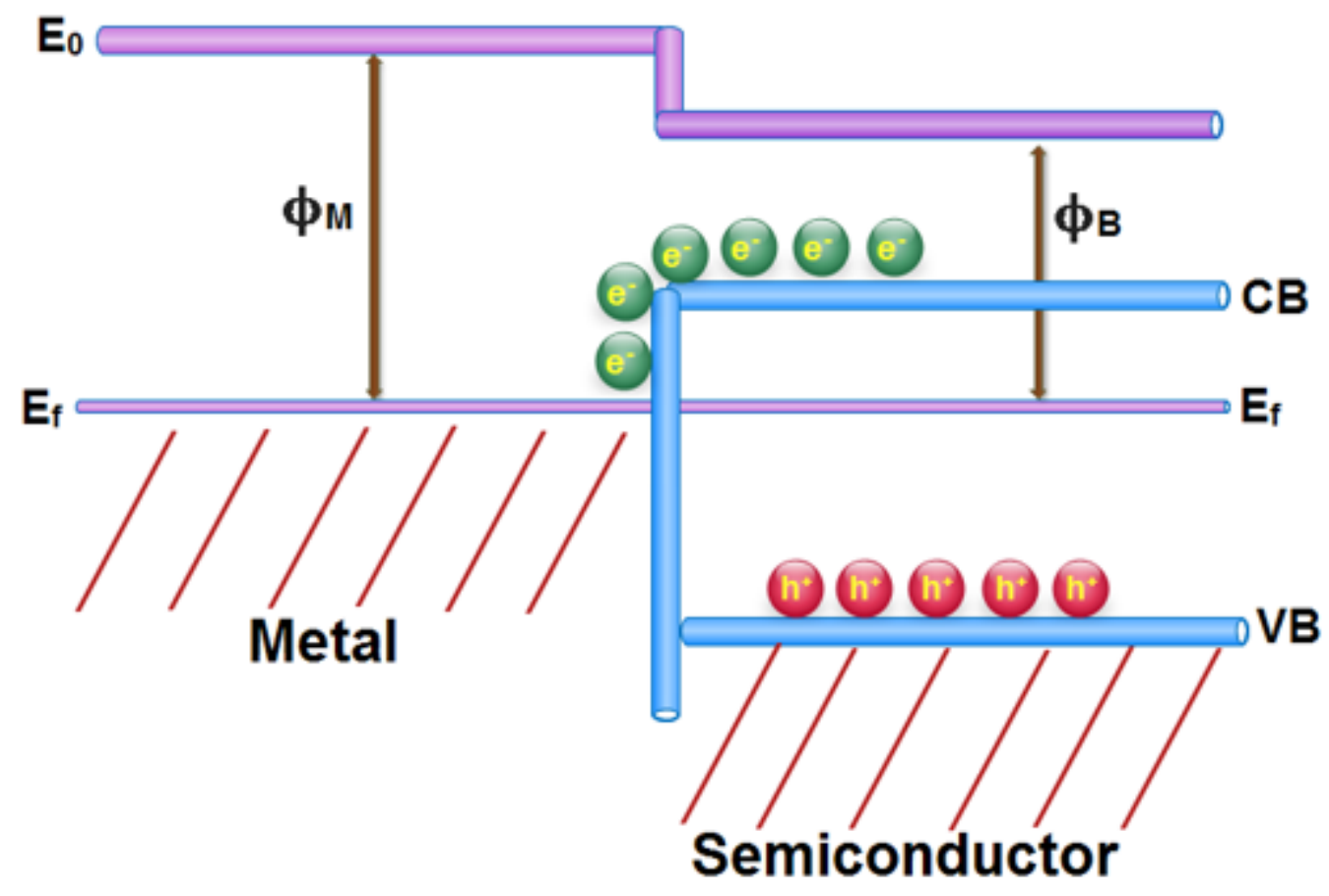

Figure 8

Qualitative electronic band structure of Ag decorated nanosheets
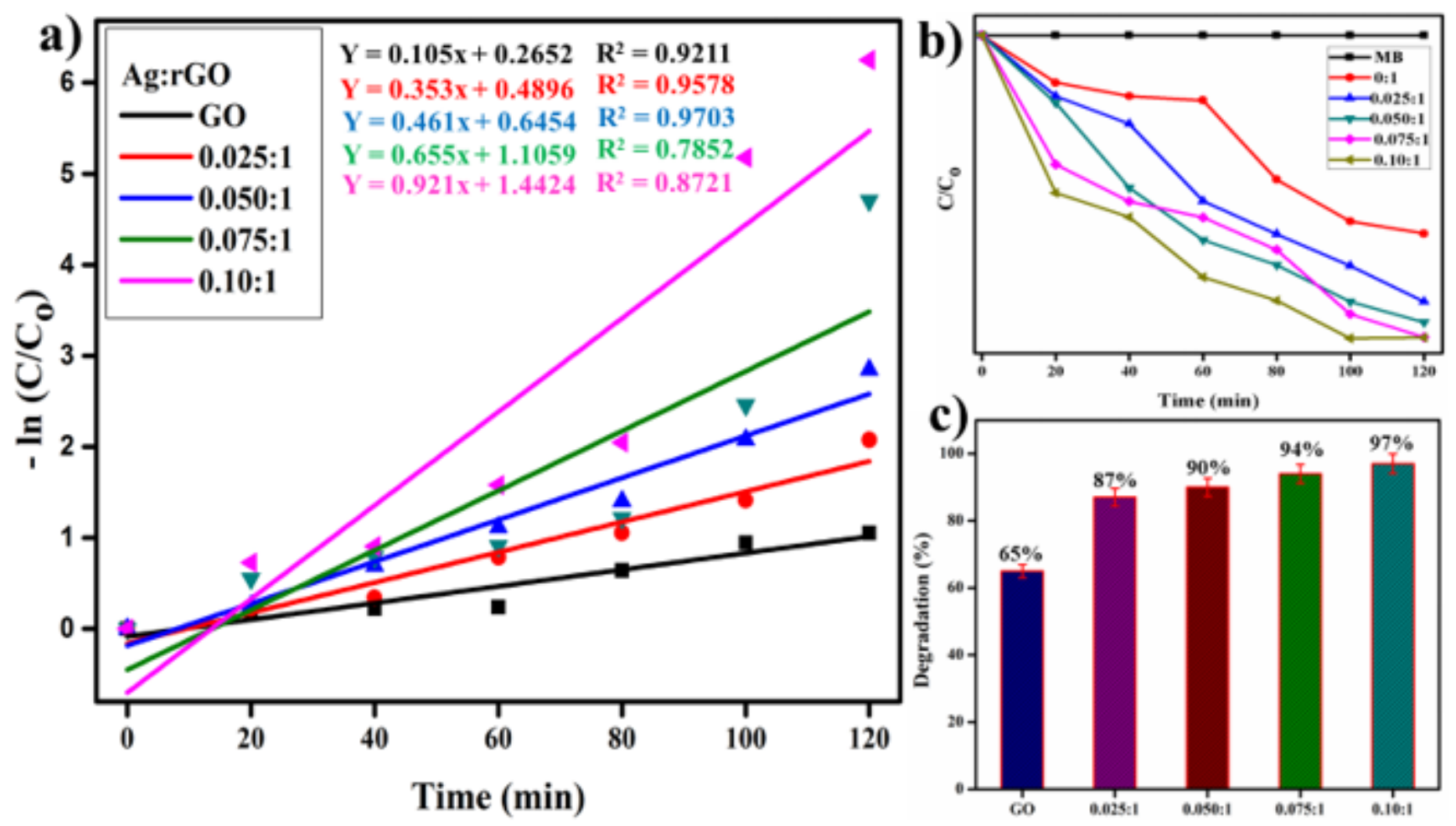

Figure 9 
(a) Plot of $-\ln (\mathrm{Ct} / \mathrm{CO})$ versus time spectra for dye reduction (b) Plot of concentration ratio $(\mathrm{C} / \mathrm{C} 0)$ versus time (c) degradation (\%) comparison of all samples.

\section{Supplementary Files}

This is a list of supplementary files associated with this preprint. Click to download.

- Equations.docx 